\title{
Improved Spatiotemporal Resolution of Dynamic Susceptibility Contrast Perfusion MRI in Brain Tumors Using Simultaneous Multi-Slice Echo-Planar Imaging
}

\author{
(D) A. Chakhoyan, (D) K. Leu, (D)W.B. Pope, DT.F. Cloughesy, and DB.M. Ellingson
}

\begin{abstract}
SUMMARY: DSC perfusion MR imaging in brain tumors requires a trade-off between spatial and temporal resolution, resulting in less spatial coverage to meet the temporal resolution requirements for accurate relative CBV estimation. DSC-MR imaging could potentially benefit from the advantages associated with simultaneous multi-slice imaging, including increased spatiotemporal resolution. In the current article, we demonstrate how simultaneous multi-slice EPI can be used to improve DSC-MR imaging spatiotemporal resolution in patients with glioblastoma.
\end{abstract}

ABBREVIATIONS: $\mathrm{rCBV}=$ relative $\mathrm{CBV}$; SMS = simultaneous multi-slice

$D^{2}$ SC perfusion MR imaging estimates of relative CBV (rCBV) have been shown to differentiate histologic tumor grade, ${ }^{1,2}$ predict survival, and be useful for therapeutic monitoring in brain tumors $^{3}$; however, DSC requires compromises in image resolution, slice coverage, and temporal resolution. The TR must be short enough to capture the rapid passage of the contrast bolus through the blood vasculature, but at the same time, there must be enough spatial coverage to encapsulate the entire tumor or brain. Simultaneous multi-slice (SMS) $\mathrm{EPI}^{4}$ has drawn widespread attention due to the advent of highly accelerated images ${ }^{5}$ through the application of composite radiofrequency pulses to simultaneously excite multiple slice planes. ${ }^{6}$ The SMS technique excites

Received April 30, 2017; accepted after revision August 24.

From the UCLA Brain Tumor Imaging Laboratory (A.C., K.L., B.M.E.), Center for Computer Vision and Imaging Biomarkers, University of California, Los Angeles, Los Angeles, California; Departments of Radiological Sciences (A.C., K.L., W.B.P., B.M.E.), Biomedical Physics (B.M.E.), Psychiatry and Biobehavioral Sciences (B.M.E.), UCLA Brain Research Institute (T.F.C., B.M.E.), and Neurology (T.F.C.), David Geffen School of Medicine, University of California, Los Angeles, Los Angeles, California; and Department of Bioengineering (K.L., B.M.E.), Henry Samueli School of Engineering and Applied Science, and UCLA Neuro-Oncology Program (T.F.C., B.M.E.), University of California, Los Angeles, Los Angeles, California.

This work was supported by the American Cancer Society Research Scholar Grant RSG-15-003-01-CCE (B.M.E.); the National Institutes of Health/National Cancer Institute R21CA167354 (B.M.E.); an American Brain Tumor Association Research Collaboration Grant supported by Humor to Fight the Tumor, ARC1700002 (B.M.E.); a National Brain Tumor Society research grant (B.M.E.); a National Institutes of Health/National Institute of General Medical Sciences training grant, GM08042 (K.L.); and the University of California, Los Angeles Medical Scientist Training Program (K.L.).

Please address correspondence to Benjamin M. Ellingson, PhD, Departments of Radiological Sciences and Psychiatry, David Geffen School of Medicine, University of California, Los Angeles, 924 Westwood Blvd, Suite 615, Los Angeles, CA 90024; e-mail: bellingson@mednet.ucla.edu; @ben_ellingson

- Indicates open access to non-subscribers at www.ajnr.org

http://dx.doi.org/10.3174/ajnr.A5433 multiple slices physically separated across the z-axis simultaneously so that each SMS "slab" has slices acquired interleaved within that section. SMS has been shown to not have appreciable loss in SNR compared with traditional methods, ${ }^{4}$ and studies have shown that SMS is effective in reducing the acquisition time with higher spatiotemporal resolution. ${ }^{5,7,8}$ In the current article, we demonstrate the clinical utility of using SMS-EPI to increase the spatiotemporal resolution of DSC-MR imaging in patients with glioblastoma.

\section{MATERIALS AND METHODS \\ Patients}

Thirteen patients with pathologically confirmed primary glioblastoma with recurrence based on either MR imaging, clinical deterioration, and/or histology were enrolled in this prospective study, which was approved by our local ethics institutional review board (University of California, Los Angeles). All patients underwent DSC-MR imaging with conventional single-shot EPI followed by SMS-EPI, 1-2 months later.

\section{DSC-MR Imaging Acquisition}

A preload bolus of $0.025 \mathrm{mmol} / \mathrm{kg}$ of Gd-DTPA (Magnevist; Bayer HealthCare Pharmaceuticals, Wayne, New Jersey) was administered to reduce leakage effects followed by $0.075 \mathrm{mmol} / \mathrm{kg}$ of Gd-DTPA at a rate of $5 \mathrm{~mL} / \mathrm{s}$. Two minutes of data were acquired, including a 30-second prebolus baseline and 90 seconds of data during and following contrast injection.

Conventional DSC-MR imaging was then acquired using a single-shot gradient-echo EPI at 3T (Prisma; Siemens, Erlangen, Germany) with $\mathrm{TE}=35 \mathrm{~ms}$, $\mathrm{TR}=1.25-1.9$ seconds, flip angle $=$ $60^{\circ}, \mathrm{FOV}=24 \times 24 \mathrm{~cm}$, matrix size $=128 \times 128$, generalized 


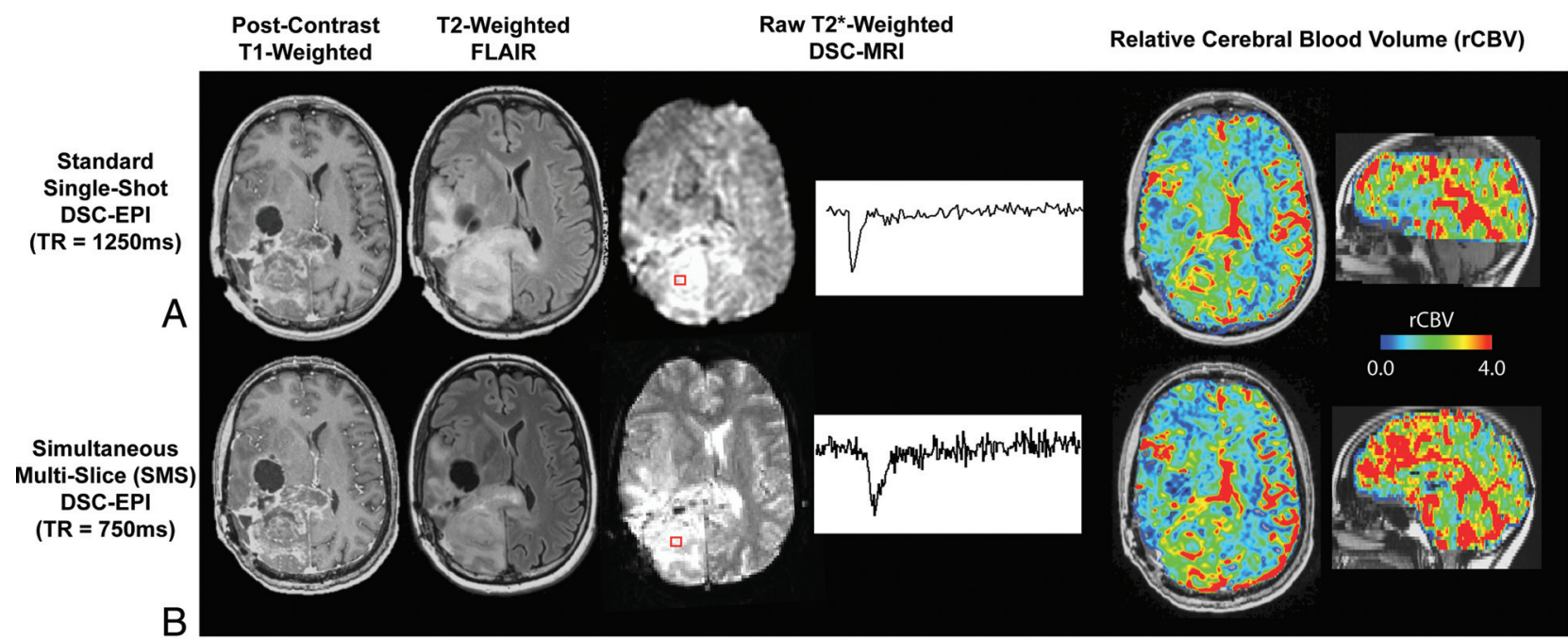

Mean Tumor CBV Differences (SMS-EPI vs. SS-EPI)

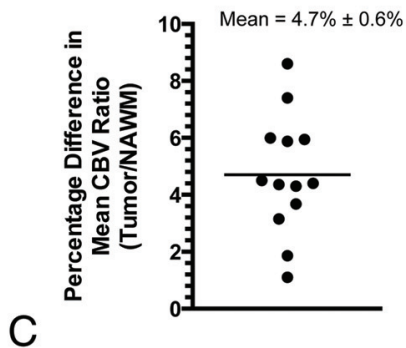

Max Tumor CBV Differences (SMS-EPI vs. SS-EPI)

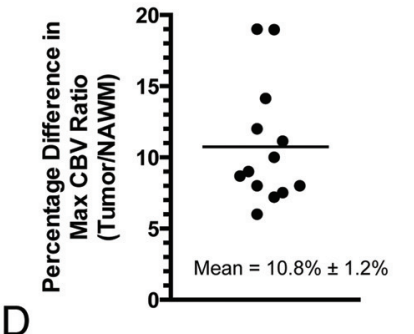

Comparison between

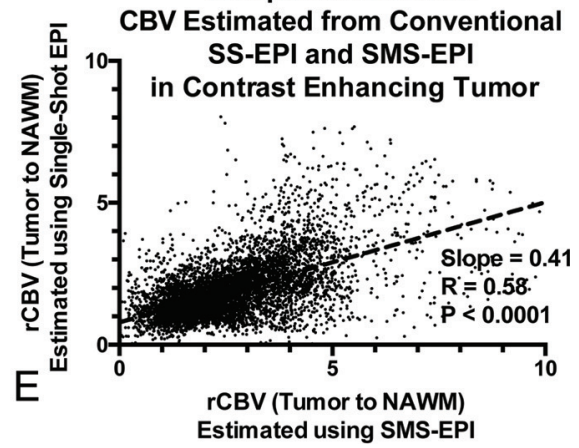

FIG 1. Postcontrast T1-weighted images, T2-weighted FLAIR images, and raw T2*-weighted DSC MR images with corresponding signal-time curves across time from tumor regions accompanied by resulting relative cerebral blood volume maps for conventional single-shot EPI $(A)$ and simultaneous multi-slice EPI ( $B$ ) (left to right). Percentage difference in mean tumor rCBV ratio (tumor to normal-appearing white matter) (C) and percentage difference in the maximum tumor rCBV ratio (tumor to normal-appearing white matter) (D) in 13 patients with recurrent glioblastoma. E, Voxelwise correlation of rCBV ratio between conventional single-shot EPI and SMS-EPI.

autocalibrating partially parallel acquisition $=2$, slice thickness $=$ $5 \mathrm{~mm}$ with no interslice gap, and 15- to 20-slice coverage. The voxel size was $1.875 \times 1.875 \times 5 \mathrm{~mm}$, and the temporal resolution was $1.25-1.90$ seconds.

Accelerated DSC-MR imaging was obtained with single-shot SMS-EPI with TE $=35 \mathrm{~ms}$, $\mathrm{TR}=750 \mathrm{~ms}$, flip angle $=60^{\circ}$, FOV $=$ $21.6 \times 24 \mathrm{~cm}$, matrix size $=180 \times 200$, slice thickness $=4 \mathrm{~mm}$ with no interslice gap, controlled aliasing in parallel imaging results in higher acceleration factor $=2$, and an SMS acceleration factor of 4 for a total of 30 slices through the whole brain. Voxel sizes were $1.2 \times 1.2 \times 4 \mathrm{~mm}$ with temporal resolution of 0.75 seconds.

\section{Postprocessing of DSC-MR Imaging}

Relative cerebral blood volume maps were generated with custom scripts including correction for bidirectional contrast agent exchange between the vascular and extravascular space. ${ }^{9}$ All rCBV values were normalized to contralateral normal-appearing white matter.

\section{ROI and Statistical Analyses}

Contrast-enhancing tumor ROIs were defined in 3D using custom scripts from Analysis of Functional Neuro Images (AFNI; http://afni.nimh.nih.gov/afni), excluding hemorrhage, large vessels, and central necrosis, followed by manual editing to exclude nonlesional voxels. ${ }^{9}$ Additionally, 2 -cm-diameter spheric ROIs were placed on contralateral normal-appearing white matter for normalization of rCBV measurements. The percentage difference in the mean $\mathrm{rCBV}$ ratio, maximum tumor $\mathrm{rCBV}$ difference, and voxelwise correlations between single-shot EPI and SMS-EPI were estimated from the resulting data for all 13 patients. GraphPad Prism software, Version 6.0h (GraphPad Software, San Diego, California) was used for all statistical analyses.

\section{RESULTS}

Both raw DSC and calculated rCBV maps were of comparable quality; however, rCBV maps calculated with SMS-EPI were obtained for the entire brain and at slightly higher spatial resolution compared with rCBV maps obtained with conventional singleshot EPI (Fig 1 $A,-B$ ). The mean tumor rCBV difference between these 2 techniques was $4.7 \% \pm 0.6 \%$ (Fig $1 C$ ), and the maximum tumor rCBV difference was $10.8 \% \pm 1.2 \%$ (Fig $1 D$ ). Voxelwise correlations in rCBV between the 2 techniques showed a significant association in all patients examined (Fig $1 E$ ), with rCBV ratios in the tumor with respect to normal-appearing white matter slightly higher when estimated using SMS-EPI. 


\section{CONCLUSIONS}

SMS-EPI can increase the spatiotemporal resolution in DSC-MR imaging, resulting in larger brain coverage (12 versus $10 \mathrm{~cm})$, higher in-plane spatial resolution $(1.2 \times 1.2 \times 4 \mathrm{~mm}$ versus $1.875 \times 1.875 \times 5 \mathrm{~mm})$, and faster temporal resolution $(0.75$ versus 1.25-1.90 seconds), with comparable results in quantification of tumor vascularity and clinical feasibility. While the potential impact on slice-to-slice perfusion quantitation is not currently known and results suggest that there may be little effect, a larger study is warranted. Together, these data demonstrate the potential clinical value of using SMS-EPI to estimate rCBV in patients with brain tumors with a high degree of reproducibility in terms of vascular biology quantification compared with historical testretest studies. ${ }^{10}$

Disclosures: Whitney B. Pope-RELATED: Consulting Fee or Honorarium: Bracco; UNRELATED: Grants/Grants Pending: Guerbet.* Benjamin M. Ellingson—RELATED: Grant: Siemens; UNRELATED: Board Membership: MedQIA, Medicenna, Roche, Agios Pharmaceuticals, Bristol-Myers Squib; Consultancy: MedQIA, Medicenna, Roche, Agios Pharmaceuticals, Bristol-Myers Squib. *Money paid to the institution.

\section{REFERENCES}

1. Aronen HJ, Gazit IE, Louis DN, et al. Cerebral blood volume maps of gliomas: comparison with tumor grade and histologic findings. $R a-$ diology 1994;191:41-51 CrossRef Medline
2. Cha S, Knopp EA, Johnson G, et al. Intracranial mass lesions: dynamic contrast-enhanced susceptibility-weighted echo-planar perfusion MR imaging. Radiology 2002;223:11-29 CrossRef Medline

3. Law M, Yang S, Wang H, et al. Glioma grading: sensitivity, specificity, and predictive values of perfusion MR imaging and proton MR spectroscopic imaging compared with conventional MR imaging. AJNR Am J Neuroradiol 2003;24:1989-98 Medline

4. Barth M, Breuer F, Koopmans PJ, et al. Simultaneous multislice (SMS) imaging techniques. Magn Reson Med 2016;75:63-81 CrossRef Medline

5. Feinberg DA, Moeller S, Smith SM, et al. Multiplexed echo planar imaging for sub-second whole brain FMRI and fast diffusion imaging. PLoS One 2010;5:e15710 CrossRef Medline

6. Maudsley AA. Multiple-line-scanning spin density imaging. J Magn Reson 1980;41:112-26

7. Chen L, T Vu A, Xu J, et al. Evaluation of highly accelerated simultaneous multi-slice EPI for fMRI. Neuroimage 2015;104:452-59 CrossRef Medline

8. Feinberg DA, Setsompop K. Ultra-fast MRI of the human brain with simultaneous multi-slice imaging. J Magn Reson 2013;229:90-100 CrossRef Medline

9. Ellingson BM, Cloughesy TF, Lai A, et al. Quantitative volumetric analysis of conventional MRI response in recurrent glioblastoma treated with bevacizumab. Neuro Oncol 2011;13:401-09 CrossRef Medline

10. Henry ME, Kaufman MJ, Lange N, et al. Test-retest reliability of DSC MRI CBV mapping in healthy volunteers. Neuroreport 2001;12: 1567-69 CrossRef Medline 